OPEN ACCESS

Edited by:

Herbert Leonel de Matos Guedes, Federal University of

Rio de Janeiro, Brazil

Reviewed by:

Tarek Adnan Ahmad,

Bibliotheca Alexandrina,

Egypt

Fengyi Jin,

University of New

South Wales, Australia

Jimmy Thomas Efird,

East Carolina University,

United States

${ }^{*}$ Correspondence:

Sally N. Adebamowo sadebamowo@som.umaryland.edu

Specialty section:

This article was submitted to Infectious Diseases - Surveillance,

Prevention and Treatment,

a section of the journal

Frontiers in Public Health

Received: 01 April 2017

Accepted: 03 July 2017

Published: 21 July 2017

Citation:

Adebamowo SN, Olawande O, Famooto A, Dareng EO, Offiong $R$

and Adebamowo CA (2017) Persistent Low-Risk and High-Risk Human Papillomavirus Infections of the Uterine Cervix in HIV-Negative and HIV-Positive Women.

Front. Public Health 5:178. doi: 10.3389/fpubh.2017.00178

\section{Persistent Low-Risk and High-Risk Human Papillomavirus Infections of the Uterine Cervix in HIV-Negative and HIV-Positive Women}

\author{
Sally N. Adebamowo ${ }^{1,2,3 *}$, Oluwatoyosi Olawande ${ }^{4}$, Ayotunde Famooto ${ }^{3,4}$, \\ Eileen O. Dareng ${ }^{5}$, Richard Offiong ${ }^{6}$ and Clement A. Adebamowo $0^{1,2,3,7}$ \\ for the H3Africa ACCME Research Group ${ }^{8}$
}

\begin{abstract}
'Department of Epidemiology and Public Health, University of Maryland School of Medicine, Baltimore, MD, United States, 2 University of Maryland Comprehensive Cancer Center, University of Maryland School of Medicine, Baltimore, MD, United States, ${ }^{3}$ Center for Bioethics and Research, Ibadan, Nigeria, ${ }^{4}$ Office of Strategic Information and Research, Institute of Human Virology Nigeria, Abuja, Nigeria, ${ }^{5}$ Department of Public Health and Primary Care, University of Cambridge, Cambridge, United Kingdom, ${ }^{6}$ Department of Obstetrics and Gynecology, University of Abuja Teaching Hospital, Abuja, Nigeria, ${ }^{7}$ Institute of Human Virology, University of Maryland School of Medicine, Baltimore, United States, ${ }^{8}$ Institute of Human Virology Nigeria, Abuja, Nigeria
\end{abstract}

Background: The prevalence, persistence, and multiplicity of human papillomavirus (HPV) infection appears different comparing HIV-positive to HIV-negative women. In this study, we examined prevalent, persistent, and multiple low- and high-risk cervical HPV infections in HIV-negative and HIV-positive women.

Methods: We studied 1,020 women involved in a study of HPV infection using $\mathrm{SPF}_{25} /$ $\mathrm{LiPA}_{10}$. Two study visits were scheduled, at enrollment and 6 months afterward. At each study visit, research nurses used a cervical brush to collect samples of exfoliated cervical cells from the cervical os, from all the study participants. Exact logistic regression models were used to estimate associations between HIV and HPV infections.

Results: The mean (SD) age of the study participants was 38 (8) years, 56\% were HIV-negative and 44\% were HIV-positive. Among HIV-negative women at baseline, single low-risk HPV (IrHPV) infections occurred in 12\%; multiple IrHPV in 2\%; single high-risk human papillomavirus (hrHPV) infections in 9\%, and multiple hrHPV infections in 2\%. Single IrHPV infections were persistent in 6\%, but there was no persistent multiple IrHPV infections. Single hrHPV infections were persistent in $4 \%$ while multiple hrHPV infections were persistent in $0.3 \%$. Among HIV-positive women at baseline, single IrHPV infections occurred in 19\%, multiple IrHPV in 6\%, single hrHPV infections in $17 \%$, and multiple hrHPV infections occurred in 12\%. Single IrHPV infections were persistent in 9\%, multiple IrHPV infections in 0.6\%, single hrHPV infections in 13\%, while multiple hrHPV were persistent in $3 \%$. Prevalent, persistent, and multiple infections were more common in HIV-positive women, compared to HIV-negative women. In multivariate models adjusted for age, marital status, socioeconomic status, age at sexual initiation, and douching, the odds ratios comparing HIV-positive to HIVnegative women, were 2.09 (95\% Cl 1.47-2.97, $p<0.001)$ for prevalent IrHPV, 1.26 
(95\% Cl 0.66-2.40, p 0.47) for persistent IrHPV infections, 3.38 (95\% Cl 2.34-4.87, $p<0.001)$ for prevalent hrHPV, and 4.49 (95\% Cl 2.26-8.91, $p<0.001)$ for persistent hrHPV infections.

Conclusion: HIV infection was associated with higher prevalence of IrHPV, hrHPV, and persistence hrHPV infections, but not persistent IrHPV infections.

Keywords: human papillomavirus, HIV, Nigeria, cervical cancer, prevalence, persistence and multiple infections

\section{INTRODUCTION}

Persistent high-risk human papillomavirus (hrHPV) infection of the uterine cervix is a necessary cause of cervical cancer. hrHPV infection is highly prevalent with some $80 \%$ of women being infected at some point in their lives (1). These infections typically start in early adulthood, shortly after sexual initiation, but their prevalence falls rapidly and the infection persists in only approximately $10 \%$ of women older than 30 years of age. Cervical cancer occurs in a small proportion (12\%) of women who have persistent hrHPV infections. Most research on hrHPV infections in Sub-Saharan Africa (SSA) to date have focused on reporting prevalent infections whose association with cervical carcinogenesis is weak, rather than persistent infections. This is due to challenges with participants' follow-up and lack of sustained systematic human papillomavirus (HPV) DNA tests'based screening programs.

Previous studies suggest that there is greater heterogeneity of HPV types in populations with high prevalence of HPV infections, thereby reducing the relative prevalence of HPV types 16 and 18 (2). The most commonly reported hrHPV types in SSA are HPV types 35,52 , and $58(3-8)$. There is a dearth of data on the risk of prevalent and persistent, single, and multiple infections with hrHPV among HIV-negative and -positive women, especially from West Africa.

Low-risk HPV (lrHPV) infections are similarly ubiquitous and are associated with common, flat plane, anogenital warts, and oral lesions (9). These diseases cause significant morbidity, are difficult to treat, and have high health systems' costs; nevertheless, there are very few studies of lrHPV in SSA (10, 11). Furthermore, little is known about the prevalence and persistence of combined lrHPV and hrHPV infections in SSA or whether interactions between lrHPV and hrHPV infections are associated with persistence of either infections. More research on prevalence, persistence, and multiplicity of HPV infections of the uterine cervix in SSA women is urgently required in order to ascertain the role of different HPV types in cervical cancer in this population.

HIV infection is associated with increased risk of cervical cancer. Several studies have shown that HIV-positive women are more likely to have prevalent and multiple HPV infections, compared to HIV-negative women $(3,4,8,12)$. Some studies show that the type distribution of HPV in HIV-positive women is different from that in HIV-negative women $(13,14)$ but not all studies support this finding (4). More studies examining the distribution of HPV infections of the uterine cervix by HIV status are warranted. The results of such studies would contribute to understanding HIV-associated cervical cancer and choice of cervical cancer prevention in people living with HIV/ AIDS (PLWA).

In this study, we examined the prevalence, persistence, and multiplicity of infections of the uterine cervix by lrHPV and hrHPV types among HIV-negative and HIV-positive women in Nigeria.

\section{MATERIALS AND METHODS}

\section{Study Population}

We studied 1,020 women who were enrolled in a study of HPV infection and cervical cancer at National Hospital, Abuja and University of Abuja Teaching Hospital, Nigeria, between 2012 and 2014. All study participants were 18 years or older, had history of prior vaginal sex, were not pregnant, and had an intact uterus at enrollment. Interviewers used questionnaires to collect data on sociodemographic characteristics, sexual and reproductive history, and HIV status, which were confirmed from medical records. During the baseline visit and the followup visit, which was scheduled to occur 6 months after enrollment, research nurses used a cervical brush to collect samples of exfoliated cervical cells from the cervical os, from all the study participants.

\section{HPV Detection by $\mathrm{SPF}_{10} / \mathrm{LiPA}_{25}$}

We extracted DNA from cervical exfoliated cells as previously described (15). Samples were tested for the presence of HPV DNA by hybridization of $\mathrm{SPF}_{10}$ amplimers to a mixture of general HPV probes recognizing a broad range of high-risk, low-risk, and possible hrHPV genotypes in a microtiter plate format, as described previously (16). All samples determined to be HPV DNA positive by $\mathrm{SPF}_{10}$ DNA enzyme immunoassay were genotyped using the $\mathrm{LiPA}_{25}$ version 1 . The $\mathrm{LiPA}_{25}$ assay provides type-specific information for 25 different HPV genotypes simultaneously and identifies infection by one or more of 13 hrHPV genotypes: 16, 18, 31, 33, 35, 39, 45, 51, 52, $56,58,59$, and $68(17,18)$. However, the test does not differentiate between HPV 68 and 73, so we defined this HPV genotype, HPV68/73, as low-risk. The test can identify lrHPV types 6,11 , $34,40,42,43,44,53,54,66,70$, and 74 . The test also identifies unspecified HPV genotypes, HPV U, which we defined as low risk. We defined HPV infection as prevalent if at least one HPV genotype was detected by the $\mathrm{SPF}_{10} / \mathrm{LiPA}_{25}$ test in a sample provided at the baseline visit; and persistent if at least one $\mathrm{HPV}$ genotype was detected by the $\mathrm{SPF}_{10} / \mathrm{LiPA}_{25}$ test in each of the samples provided at consecutive baseline and followup visits. 


\section{Statistical Analysis}

In order to compute socioeconomic status (SES) in a low resource environment where income data is sparse, we generated wealth index data as previously described (19). In summary, we used principal components analysis with varimax rotation to compute factor scores based on the sum of the ownership of household items weighted by their factor loading. We sorted the data on the first principal component, which had the highest eigenvalue and divided all respondents into three categories based on its value. Participants in the lowest $40 \%$ were categorized as low SES, the middle $40 \%$ were categorized as middle SES, and the top 20\% were categorized as high SES. The validity and reproducibility of wealth index has been examined in previous studies and it correlates well with other measures of wealth in environments without reliable expenditure data (19).

We used $t$-tests to assess differences in the distribution of continuous variables between groups and $\chi^{2}$ and Fisher's exact tests for categorical variables. We used exact logistic regression models to evaluate factors associated with HPV infection and between HIV and HPV infections in the study population $(20,21)$. We conducted age-adjusted analyses of demographic and behavioral characteristics of the participants and their associations with HPV infections. We selected those variables that were associated at $p<0.20$ for inclusion in multivariate models. All the $p$-values reported were two-sided. All analyses were performed using SAS 9.3 for UNIX statistical software (SAS Institute, Cary, NC, USA).

\section{Ethics}

The study was conducted according to the Nigerian National Code for Health Research Ethics. Ethical approval to conduct this study was obtained from the Institute of Human Virology Nigeria research ethics committee. Written informed consent was obtained from all participants before enrollment in the study, in accordance with the Declaration of Helsinki.

\section{RESULTS}

Of the 1,020 participants enrolled at baseline, we excluded 58 participants due to missing results (41 missing HIV results, 13 missing baseline HPV results, and 4 missing both HIV and baseline HPV results) leaving 962 women in the study. Some $72 \%(692 / 962)$ of these women completed follow-up visits. Of these, we excluded 62 participants whose HPV results were missing at follow-up leaving 630 women who had complete data for analysis for persistent HPV infections. The median time interval between the baseline and follow-up visits was 9 months. The participants' flow through the study is depicted in Figure 1.

\section{HIV-Negative Women}

Most of the women $(56 \%, 535 / 962)$ were HIV-negative at baseline. The mean (SD) age of the HIV-negative women was 38 (8) years while their mean (SD) body mass index (BMI) (kilogram per square meter) was 29.0 (8.5). The characteristics of the study participants at baseline are shown in Table $\mathbf{1}$.

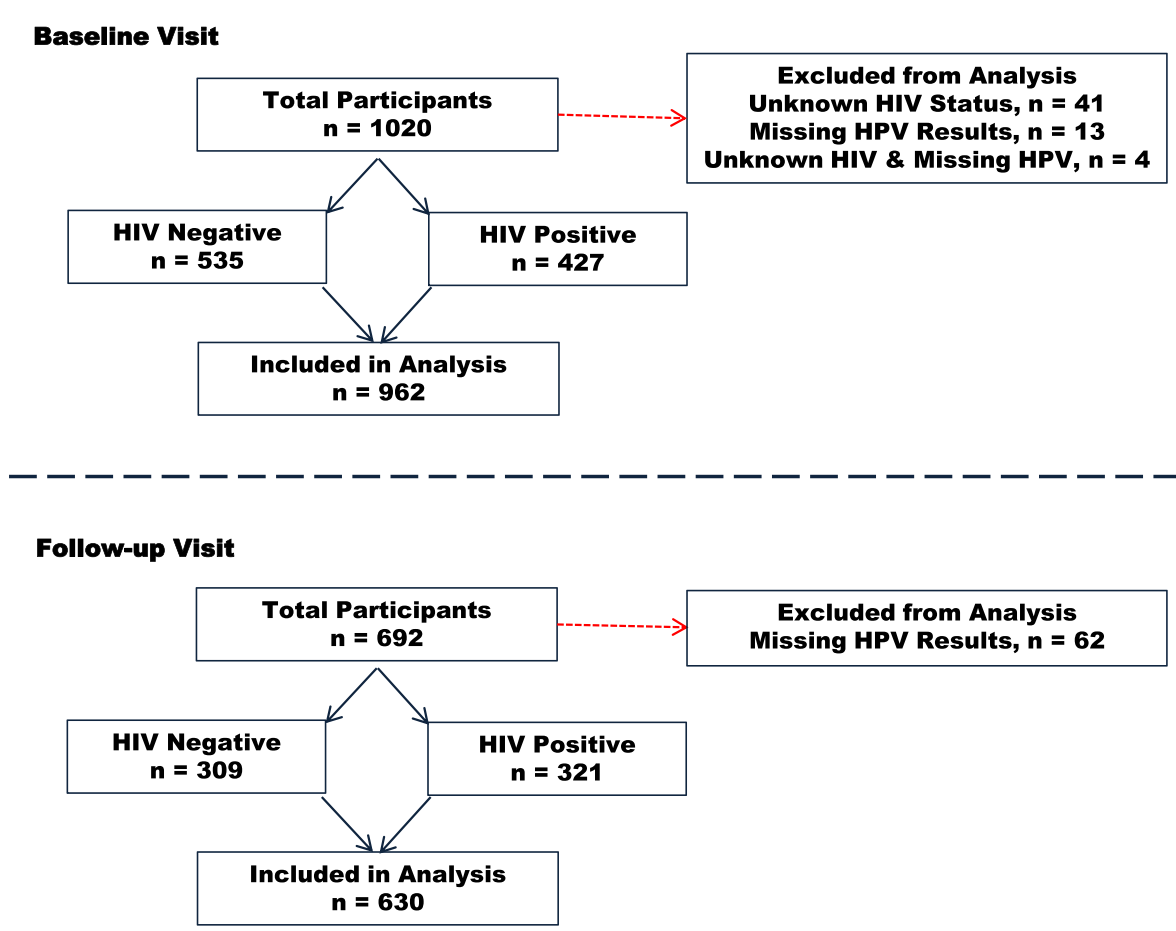

FIGURE 1 | Participant flow chart. 
TABLE 1 | Baseline characteristics of the study population.

\begin{tabular}{|c|c|c|c|}
\hline Characteristics & $\begin{array}{c}\text { Total } \\
n=962\end{array}$ & $\begin{array}{c}\text { HIV-negative } \\
n=535\end{array}$ & $\begin{array}{c}\text { HIV-positive } \\
n=427\end{array}$ \\
\hline & \multicolumn{3}{|c|}{ Mean (SD) } \\
\hline Age, years & $38( \pm 8)$ & $38(8)$ & $37(7)$ \\
\hline Body mass index (BMI), $\mathrm{kg} / \mathrm{m}^{2}$ & $28.1(11.9)$ & $29.0(8.5)$ & $26.9(15.2)$ \\
\hline Age at sexual initiation, years & $20( \pm 4)$ & $21(4)$ & $19(3)$ \\
\hline \multirow[t]{2}{*}{ Total sex partners } & $3( \pm 3)$ & $3(2)$ & $4(4)$ \\
\hline & \multicolumn{3}{|c|}{$n(\%)$} \\
\hline \multicolumn{4}{|l|}{ Age, years } \\
\hline $18-29$ & $127(13)$ & $72(13)$ & $55(13)$ \\
\hline 30-39 & $447(47)$ & $222(42)$ & $225(53)$ \\
\hline $40-49$ & 317 (33) & $196(37)$ & $121(28)$ \\
\hline$\geq 50$ & $71(7)$ & $45(8)$ & $26(6)$ \\
\hline \multicolumn{4}{|l|}{ BMI, kg/m2 } \\
\hline Normal weight, 18.5-24.9 & $303(33)$ & $136(27)$ & $167(42)$ \\
\hline Overweight, 25.0-29.9 & $324(36)$ & $184(36)$ & $240(35)$ \\
\hline Obese, $\geq 30.0$ & $282(31)$ & $192(37)$ & $90(23)$ \\
\hline \multicolumn{4}{|l|}{ Marital status } \\
\hline Married & $647(67)$ & $421(79)$ & $226(53)$ \\
\hline Not married & $315(33)$ & $114(21)$ & $201(47)$ \\
\hline \multicolumn{4}{|l|}{ Education } \\
\hline$\leq 6$ years & $104(11)$ & $45(8)$ & $59(14)$ \\
\hline Secondary & $602(63)$ & $294(55)$ & $308(72)$ \\
\hline Tertiary & $254(26)$ & $195(37)$ & $59(14)$ \\
\hline \multicolumn{4}{|l|}{ Socioeconomic status } \\
\hline Low & $375(39)$ & $152(28)$ & $223(52)$ \\
\hline Middle & $384(40)$ & $226(43)$ & $158(37)$ \\
\hline High & $203(21)$ & $157(29)$ & $46(11)$ \\
\hline \multicolumn{4}{|c|}{ Age at sexual initiation, years } \\
\hline$<18$ & $216(23)$ & $95(18)$ & $121(29)$ \\
\hline $18-20$ & $377(40)$ & $196(47)$ & $181(44)$ \\
\hline $21-23$ & $180(19)$ & $113(21)$ & $67(16)$ \\
\hline$>24$ & $174(18)$ & $127(24)$ & $47(11)$ \\
\hline \multicolumn{4}{|l|}{ Total sex partners } \\
\hline 1 & $249(26)$ & $179(33)$ & $70(16)$ \\
\hline $2-3$ & $393(41)$ & $217(41)$ & $176(41)$ \\
\hline $4-5$ & $197(21)$ & $91(17)$ & $106(16)$ \\
\hline$>5$ & $119(12)$ & $47(9)$ & $72(17)$ \\
\hline \multicolumn{4}{|l|}{ Contraceptive use } \\
\hline Yes & $405(42)$ & $266(50)$ & $139(32)$ \\
\hline No & $557(58)$ & $269(50)$ & $288(68)$ \\
\hline \multicolumn{4}{|l|}{ Vaginal $\mathrm{pH}$} \\
\hline$<4.5$ & $73(8)$ & $47(9)$ & $26(6)$ \\
\hline $4.5-5.5$ & $68(7)$ & $34(6)$ & $34(8)$ \\
\hline$>5.5$ & $821(85)$ & $454(85)$ & $367(86)$ \\
\hline \multicolumn{4}{|l|}{ Menopausal status } \\
\hline Premenopausal & $813(85)$ & $443(84)$ & $370(87)$ \\
\hline Postmenopausal & $140(15)$ & $86(16)$ & $54(13)$ \\
\hline \multicolumn{4}{|l|}{ Douching } \\
\hline Yes & $606(63)$ & $319(60)$ & $287(67)$ \\
\hline No & $355(37)$ & $216(40)$ & 139 (33) \\
\hline
\end{tabular}

Presence of any type of HPV infection of the cervix was detected in $22 \%(114 / 535)$ of these HIV-negative women at baseline, while $10 \%(31 / 309)$ had persistence of any type of HPV infections of the uterine cervix. The infection involved a single HPV type at baseline in 17\% (91/535) of these women and there was persistence of any type of HPV infection in 10\% (30/309). Multiple infections by any type of HPV were present in $4.3 \%$ $(23 / 535)$ at baseline but only $0.3 \%(1 / 309)$ had persistent multiple HPV infections (Table 2).
TABLE 2 | Prevalence of human papillomavirus (HPV) infections in the study population, $n(\%)$.

\begin{tabular}{|c|c|c|c|c|}
\hline Prevalent infections & $\begin{array}{c}\text { All } \\
n=962\end{array}$ & $\begin{array}{c}\text { HIV-negative } \\
n=535\end{array}$ & $\begin{array}{c}\text { HIV-positive } \\
n=427\end{array}$ & $p$-Value \\
\hline \multicolumn{5}{|c|}{ All prevalent HPV infections } \\
\hline Any HPV & $300(31.2)$ & $114(21.3)$ & $186(43.5)$ & $<0.001$ \\
\hline Low risk & $181(18.8)$ & $72(13.5)$ & $109(25.5)$ & $<0.001$ \\
\hline High risk & $181(18.8)$ & $57(10.6)$ & $124(29.0)$ & $<0.001$ \\
\hline \multicolumn{5}{|l|}{ Single prevalent HPV } \\
\hline Any HPV & 191 (19.6) & $91(17.0)$ & $100(23.4)$ & 0.01 \\
\hline Low risk & $145(15.1)$ & $63(11.9)$ & $82(19.2)$ & 0.001 \\
\hline High risk & $119(12.4)$ & $47(8.8)$ & $72(16.9)$ & $<0.001$ \\
\hline \multicolumn{5}{|l|}{ Multiple prevalent HPV } \\
\hline Any HPV & 109 (11.3) & $23(4.3)$ & $86(20.1)$ & $<0.001$ \\
\hline Low risk & $36(3.7)$ & $9(1.7)$ & $27(6.3)$ & $<0.001$ \\
\hline High risk & $62(6.4)$ & $10(1.9)$ & $52(12.2)$ & $<0.001$ \\
\hline Persistent infections & $\begin{array}{c}\text { All } \\
n=630\end{array}$ & $\begin{array}{l}\text { HIV-negative } \\
\qquad n=309\end{array}$ & $\begin{array}{c}\text { HIV-positive } \\
n=321\end{array}$ & \\
\hline \multicolumn{5}{|c|}{ All persistent HPV infections } \\
\hline Any HPV & 107 (16.9) & $31(10.0)$ & $76(23.7)$ & $<0.001$ \\
\hline Low risk & $50(7.9)$ & $19(6.2)$ & $31(9.7)$ & 0.10 \\
\hline High risk & $63(10.0)$ & $12(3.9)$ & $51(15.9)$ & $<0.001$ \\
\hline \multicolumn{5}{|l|}{ Single persistent HPV } \\
\hline Any HPV & $92(14.6)$ & $30(9.7)$ & $62(19.3)$ & 0.001 \\
\hline Low risk & $48(7.6)$ & $19(6.2)$ & $29(9.0)$ & 0.18 \\
\hline High risk & $53(8.4)$ & $11(3.6)$ & $42(13.1)$ & $<0.001$ \\
\hline \multicolumn{5}{|c|}{ Multiple persistent HPV } \\
\hline Any HPV & $15(2.4)$ & $1(0.3)$ & $14(4.4)$ & $<0.001$ \\
\hline Low risk & $2(0.3)$ & $0(0.0)$ & $2(0.6)$ & 0.49 \\
\hline High risk & $10(1.6)$ & $1(0.3)$ & $9(2.8)$ & 0.02 \\
\hline
\end{tabular}

Single lrHPV infections were present in 12\% (63/535) and multiple lrHPV infections were present in $2 \%$ (9/535) of the women at baseline, while single hrHPV infections were present in $9 \%(47 / 535)$ of the women and multiple hrHPV infections were present in $2 \%(10 / 535)$ of the women at baseline. Single lrHPV infections were persistent in 6\% (19/309), but we did not observe persistent infections with multiple lrHPV types. Single hrHPV infections were persistent in 4\% (11/309) of the women and multiple hrHPV infections were persistent in $0.3 \%(1 / 309)$ of the women.

The commonest prevalent lrHPV types detected in these HIVnegative women were unspecified HPV types $(31 / 535,5.8 \%)$, HPV53 (10/535, 1.9\%), and HPV68/73 (10/535, 1.9\%) while the commonest persistent lrHPV types were unspecified HPV types (7/309, 2.3\%) and HPV 68/73 (5/309, 1.6\%). The commonest prevalent hrHPV types were HPV52 (16/535, 3.0\%) and HPV18 $(9 / 535,1.7 \%)$. HPV 16 was detected in only $0.8 \%$ (4/535) of these women at baseline (Table 3), while the commonest persistent hrHPV types were HPV52 (5/309, 1.6\%) and HPV 35 (3/309, $1.0 \%)$. We did not find any persistent infections with HPV 16 or HPV18 (Table 4).

\section{HIV-Positive Women}

Some 44\% (427/962) of the women were HIV-positive at baseline. The mean (SD) age of the HIV-positive women was 37 (7) years while their mean (SD) BMI (kilogram per square meter) was 26.9 (5.2). 
TABLE 3 | Prevalence and persistence of type-specific low-risk human papillomavirus (HPV) in the total study population and by HIV status, $n$ (\%).

\begin{tabular}{|c|c|c|c|c|c|c|c|c|}
\hline \multirow[t]{3}{*}{ HPV type } & \multicolumn{4}{|c|}{ Prevalent HPV } & \multicolumn{4}{|c|}{ Persistent HPV } \\
\hline & Total & HIV-negative & HIV-positive & $p$-Value & Total & HIV-negative & HIV-positive & $p$-Value \\
\hline & $n=962$ & $n=535$ & $n=427$ & & $n=630$ & $n=309$ & $n=321$ & \\
\hline HPV 6 & $10(1.0)$ & $4(0.8)$ & $6(1.4)$ & 0.35 & $1(0.2)$ & $0(0.0)$ & $1(0.3)$ & 1.00 \\
\hline HPV 11 & $15(1.6)$ & $6(1.1)$ & $9(2.1)$ & 0.29 & $2(0.3)$ & $1(0.3)$ & $1(0.3)$ & 1.00 \\
\hline HPV 34 & $1(0.1)$ & $0(0.0)$ & $1(0.2)$ & 0.44 & $0(0.0)$ & $0(0.0)$ & $0(0.0)$ & - \\
\hline HPV 40 & $4(0.4)$ & $1(0.2)$ & $3(0.7)$ & 0.32 & $0(0.0)$ & $0(0.0)$ & $0(0.0)$ & - \\
\hline HPV 42 & $3(0.3)$ & $0(0.0)$ & $3(0.7)$ & 0.08 & $1(0.2)$ & $0(0.0)$ & $1(0.3)$ & 1.00 \\
\hline HPV 43 & $6(0.6)$ & $2(0.4)$ & $4(0.9)$ & 0.41 & $2(0.3)$ & $0(0.0)$ & $2(0.6)$ & 0.49 \\
\hline HPV 44 & $22(2.3)$ & $4(0.8)$ & $18(4.2)$ & $<0.01$ & $7(1.1)$ & $0(0.0)$ & $7(2.2)$ & 0.01 \\
\hline HPV 53 & $21(2.2)$ & $10(1.9)$ & $11(2.6)$ & 0.50 & $4(0.6)$ & $2(0.6)$ & $2(0.6)$ & 1.00 \\
\hline HPV 54 & $17(1.8)$ & $3(0.6)$ & $14(3.3)$ & $<0.01$ & $3(0.5)$ & $2(0.6)$ & $1(0.3)$ & 0.61 \\
\hline HPV 66 & $28(2.9)$ & $5(0.9)$ & $23(5.4)$ & $<0.01$ & $9(1.4)$ & $1(0.3)$ & $8(2.5)$ & 0.03 \\
\hline HPV 70 & $16(1.7)$ & $4(0.8)$ & $12(2.8)$ & 0.01 & $6(0.9)$ & $1(0.3)$ & $5(1.6)$ & 0.21 \\
\hline HPV 74 & $11(1.1)$ & $2(0.4)$ & $9(2.1)$ & 0.01 & $1(0.2)$ & $0(0.0)$ & $1(0.3)$ & 1.00 \\
\hline HPV 68/73 & $16(1.8)$ & $10(2.2)$ & $6(1.4)$ & 0.62 & $5(0.8)$ & $5(1.6)$ & $0(0.0)$ & 0.02 \\
\hline Unspecified (U) & $54(5.6)$ & $31(5.8)$ & $23(5.4)$ & 0.88 & $11(1.7)$ & $7(2.3)$ & $4(1.3)$ & 0.37 \\
\hline
\end{tabular}

TABLE 4 | Prevalence and persistence of type-specific high-risk human papillomavirus (HPV) in the total study population and by HIV status, $n$ (\%).

\begin{tabular}{|c|c|c|c|c|c|c|c|c|}
\hline \multirow[t]{3}{*}{ HPV type } & \multicolumn{3}{|c|}{ Prevalent HPV } & \multirow{3}{*}{$p$-Value } & \multicolumn{4}{|c|}{ Persistent HPV } \\
\hline & Total & HIV-negative & HIV-positive & & Total & HIV-negative & HIV-positive & $p$-Value \\
\hline & $n=962$ & $n=535$ & $n=427$ & & $n=630$ & $n=309$ & $n=321$ & \\
\hline HPV 16 & $17(1.8)$ & $4(0.8)$ & $13(3.0)$ & 0.01 & $4(0.6)$ & $0(0.0)$ & $4(1.3)$ & 0.12 \\
\hline HPV 18 & $31(3.2)$ & $9(1.7)$ & $22(5.1)$ & $<0.01$ & $4(0.6)$ & $0(0.0)$ & $4(1.3)$ & 0.12 \\
\hline HPV 31 & $23(2.4)$ & $4(0.8)$ & 19 (4.5) & $<0.01$ & $8(1.3)$ & $1(0.3)$ & $7(2.2)$ & 0.06 \\
\hline HPV 33 & $22(2.4)$ & $5(0.9)$ & 17 (3.9) & $<0.01$ & $4(0.6)$ & $1(0.3)$ & $3(0.9)$ & 0.62 \\
\hline HPV 35 & 37 (3.9) & $7(1.3)$ & $30(7.0)$ & $<0.01$ & $17(2.7)$ & $3(1.0)$ & $14(4.4)$ & 0.01 \\
\hline HPV 39 & $10(1.0)$ & $5(0.9)$ & $5(1.2)$ & 0.75 & $2(0.3)$ & $0(0.0)$ & $2(0.6)$ & 0.49 \\
\hline HPV 45 & $14(1.5)$ & $5(0.9)$ & $9(2.1)$ & 0.17 & $3(0.5)$ & $1(0.3)$ & $2(0.6)$ & 1.00 \\
\hline HPV 51 & $17(1.8)$ & $2(0.4)$ & $15(3.5)$ & $<0.01$ & $5(0.8)$ & $0(0.0)$ & $5(1.6)$ & 0.06 \\
\hline HPV 52 & $54(5.6)$ & $16(3.0)$ & 38 (8.9) & $<0.01$ & $22(3.5)$ & $5(1.6)$ & $17(5.3)$ & 0.01 \\
\hline HPV 56 & $17(1.8)$ & 7 (1.3) & $10(2.3)$ & 0.32 & $2(0.3)$ & $0(0.0)$ & $2(0.6)$ & 0.49 \\
\hline HPV 58 & 15 (1.6) & $4(0.8)$ & $11(2.6)$ & 0.03 & $4(0.6)$ & $2(0.7)$ & $2(0.6)$ & 1.00 \\
\hline HPV 59 & $10(1.0)$ & $0(0.0)$ & $10(2.3)$ & $<0.01$ & $1(0.2)$ & $0(0.0)$ & $1(0.3)$ & 1.00 \\
\hline
\end{tabular}

Any type of HPV infection of the cervix was detected in $44 \%$ $(186 / 427)$ of these women at baseline, while $24 \%(76 / 321)$ had persistence of any type of HPV infections of the uterine cervix. The infection involved a single HPV type at baseline in $23 \%$ $(100 / 427)$ of these women and there was persistence in $19 \%$ (62/321). Multiple infections by any type of HPV occurred in $20 \%(86 / 427)$ at baseline and this persisted in $4.4 \%(14 / 321)$ at follow-up (Table 2).

Single lrHPV infections were present in 19\% (82/427) and multiple lrHPV infections were present in 6\% (27/427) of the women at baseline, while single hrHPV infections were present in $17 \%(72 / 427)$ of the women and multiple hrHPV infections were present in 12\% (52/427) of the women at baseline. Single lrHPV infections were persistent in 9\% (29/321) and multiple lrHPV infections were persistent in $0.6 \%(2 / 321)$ of the women. Single hrHPV infections were persistent in 13\% (42/321) of the women and multiple hrHPV infections were persistent in 3\% (9/321) of the women.

The commonest prevalent lrHPV types detected in these women were unspecified HPV types (23/427, 5.4\%), HPV66
(23/427, 5.4\%), and HPV44 (18/427, 4.2\%) while the commonest persistent lrHPV types detected were HPV66 (8/321, 2.5\%) and HPV44 (7/321, 2.2\%). The commonest prevalent hrHPV types found were HPV52 (38/427, 8.9\%) and HPV35 (30/427, $7.0 \%)$ while the commonest persistent hrHPV types were HPV52 (17/321, 5.5\%) and HPV35 (14/321, 4.4\%). HPV16 was detected in only $3 \%(13 / 427)$, while HPV18 was detected in $5 \%(22 / 427)$ of these women at baseline (Table 3). HPV16 and HPV18 were persistent in $1.3 \%$ of the HIV-positive women (Table 4).

\section{Impact of HIV Infection}

HIV-positive women had higher prevalence, persistence, and presence of multiple HPV infections overall, compared to HIV-negative women (Figure 2). The prevalence of each hrHPV type found in HIV-positive women was also higher than that in HIV-negative women (Figure 3).

In univariate analyses, HIV status and SES were associated with prevalent HPV (Table 5), while HIV status and douching were associated with persistent HPV (Table 6). 


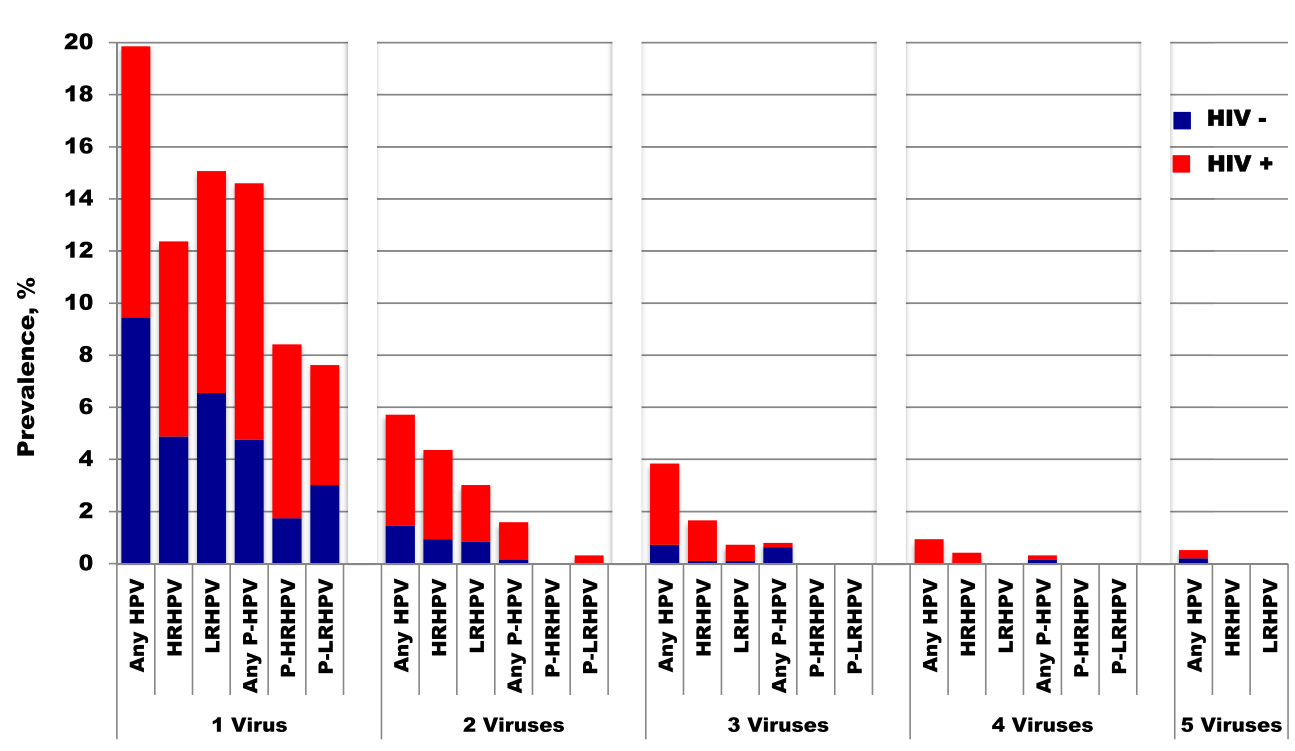

Number and Type of Virus causing HPV infection

FIGURE 2 | Prevalence of single and multiple HPV infectious by HIV status. HPV, human papillomavirus; HRHPV, high-risk HPV; LRHPV, low-risk HPV; P, persistent. One HIV-positive woman with six any genotypes was excluded from this figure.
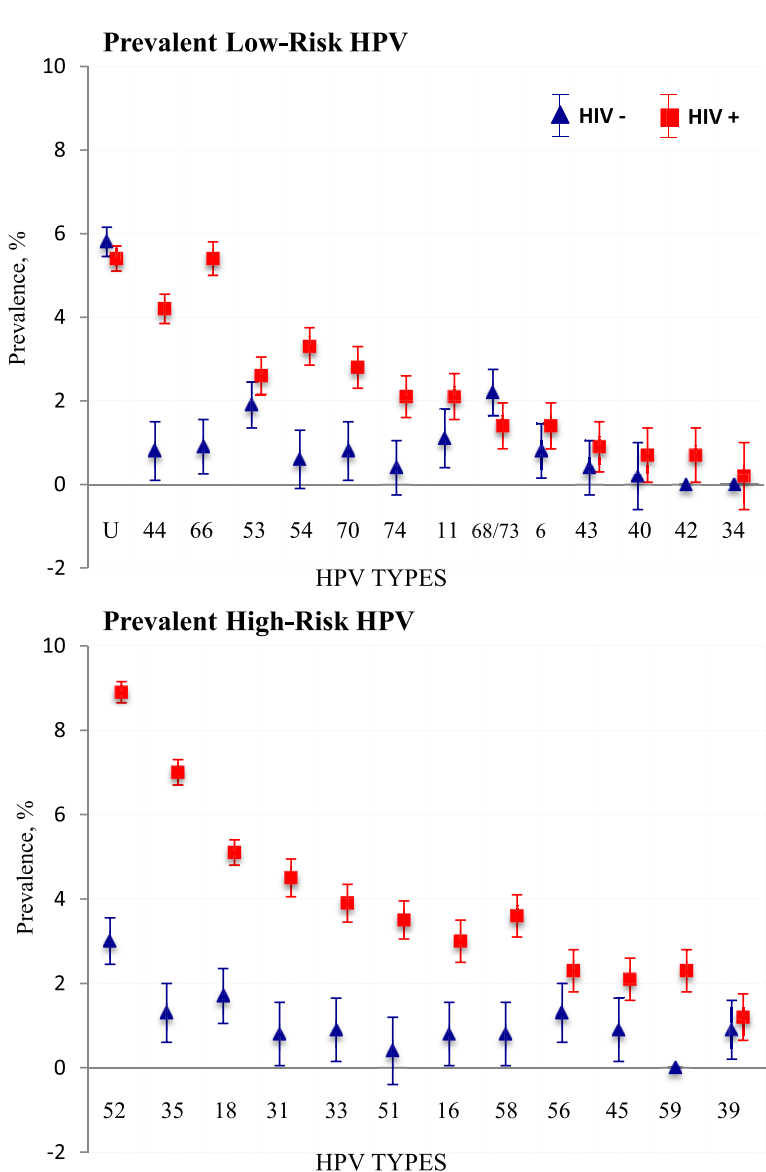
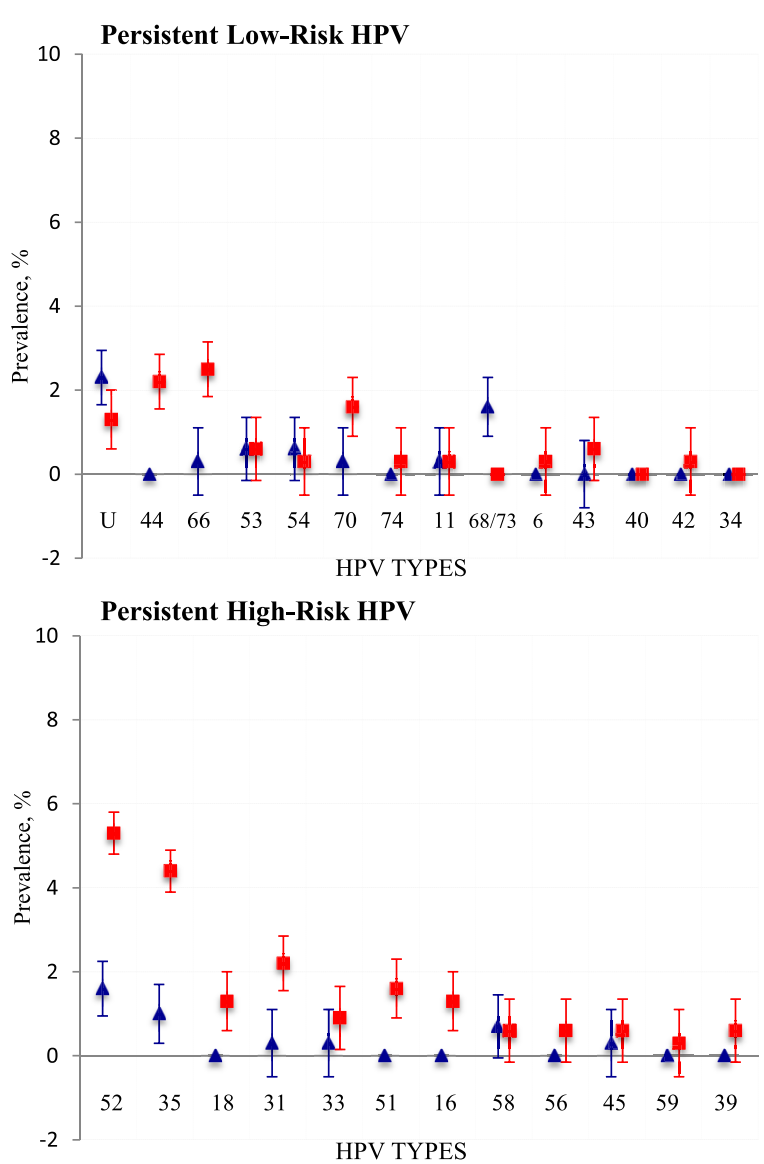

HPV TYPES

Error bars are $95 \%$ confidence intervals of the prevalence estimates

FIGURE 3 | Type-specific prevalence of human papillomavirus (HPV) infections by HIV status. Error bars are 95\% confidence intervals of prevalence estimates. 
TABLE 5 | Association between sociodemographic characteristics and potential risk factors for prevalent low-risk and high-risk human papillomavirus (HPV) infections.

\begin{tabular}{|c|c|c|c|c|c|c|c|}
\hline \multirow[t]{2}{*}{ Variable } & \multirow[t]{2}{*}{ Total $n$} & \multicolumn{3}{|c|}{ Prevalent low-risk HPV } & \multicolumn{3}{|c|}{ Prevalent high-risk HPV } \\
\hline & & $n(\%)$ & Odds ratio $(95 \% \mathrm{Cl})$ & $p$-Value & $n(\%)$ & Odds ratio $(95 \% \mathrm{Cl})$ & $p$-Value \\
\hline \multicolumn{8}{|l|}{ Age, years } \\
\hline$<30$ & 127 & $29(23)$ & $1.28(0.73-2.24)$ & 0.38 & $27(21)$ & $1.31(0.73-2.34)$ & 0.35 \\
\hline 30 to $<45$ & 659 & $119(18)$ & $0.95(0.62-1.46)$ & 0.83 & $124(19)$ & $1.12(0.72-1.74)$ & 0.59 \\
\hline$\geq 45$ & 176 & $33(19)$ & $\operatorname{Ref}(1.00)$ & & $30(17)$ & $\operatorname{Ref}(1.00)$ & \\
\hline \multicolumn{8}{|c|}{ Body mass index, $\mathrm{kg} / \mathrm{m}^{2}$} \\
\hline Normal weight & 303 & $67(22)$ & 1.38 (0.91-2.09) & 0.12 & $69(23)$ & $1.73(1.13-2.65)$ & 0.01 \\
\hline Overweight & 324 & $49(15)$ & $0.86(0.56-1.34)$ & 0.52 & $59(18)$ & $1.30(0.84-2.02)$ & 0.22 \\
\hline Obese, $\geq 30$ & 282 & $48(17)$ & $\operatorname{Ref}(1.00)$ & & $41(15)$ & $\operatorname{Ref}(1.00)$ & \\
\hline \multicolumn{8}{|l|}{ Marital status } \\
\hline Married & 647 & $114(18)$ & $0.79(0.56-1.10)$ & 0.17 & $115(18)$ & $0.81(0.58-1.14)$ & 0.23 \\
\hline Not married & 315 & $67(21)$ & $\operatorname{Ref}(1.00)$ & & $66(21)$ & $\operatorname{Ref}(1.00)$ & \\
\hline \multicolumn{8}{|c|}{ Education, years completed } \\
\hline$\leq 6$ years & 104 & $17(16)$ & $1.11(0.59-2.07)$ & 0.74 & $26(25)$ & $1.50(0.87-2.60)$ & 0.14 \\
\hline $7-12$ & 602 & $125(21)$ & $1.48(1.00-2.21)$ & 0.04 & $109(18)$ & $1.00(0.68-1.46)$ & 1.00 \\
\hline$>12$ & 254 & $38(15)$ & $\operatorname{Ref}(1.00)$ & & $46(18)$ & $\operatorname{Ref}(1.00)$ & \\
\hline \multicolumn{8}{|c|}{ Socioeconomic status } \\
\hline Low & 375 & $82(22)$ & $1.82(1.13-2.92)$ & 0.01 & $87(23)$ & $1.96(1.23-3.15)$ & $<0.01$ \\
\hline Middle & 384 & $72(19)$ & $1.50(0.93-2.42)$ & 0.09 & $67(17)$ & $1.37(0.85-2.23)$ & 0.19 \\
\hline High & 203 & $27(13)$ & $\operatorname{Ref}(1.00)$ & & $27(13)$ & $\operatorname{Ref}(1.00)$ & \\
\hline \multicolumn{8}{|c|}{ Age at sexual initiation, years } \\
\hline$\leq 19$ & 441 & $88(20)$ & $1.05(0.69-1.58)$ & 0.81 & $86(21)$ & $1.31(0.85-2.03)$ & 0.21 \\
\hline $19-22$ & 287 & $46(16)$ & $0.80(0.50-1.27)$ & 0.35 & $59(21)$ & $1.40(0.88-2.24)$ & 0.14 \\
\hline$>22$ & 219 & $42(19)$ & $\operatorname{Ref}(1.00)$ & & $34(16)$ & $\operatorname{Ref}(1.00)$ & \\
\hline \multicolumn{8}{|c|}{ Total lifetime sex partners } \\
\hline 1 & 249 & $45(18)$ & $1.04(0.65-1.69)$ & 0.84 & $34(14)$ & $0.64(0.39-1.05)$ & 0.08 \\
\hline $2-4$ & 496 & $97(20)$ & $1.15(0.76-1.75)$ & 0.49 & $104(21)$ & $1.08(0.72-1.61)$ & 0.70 \\
\hline$\geq 5$ & 213 & $37(17)$ & $\operatorname{Ref}(1.00)$ & & $42(20)$ & $\operatorname{Ref}(1.00)$ & \\
\hline \multicolumn{8}{|l|}{ Douche } \\
\hline Yes & 606 & $116(19)$ & $1.05(0.75-1.47)$ & 0.75 & $119(20)$ & $1.15(0.82-1.62)$ & 0.40 \\
\hline No & 355 & $65(18)$ & $\operatorname{Ref}(1.00)$ & & $62(17)$ & $\operatorname{Ref}(1.00)$ & \\
\hline \multicolumn{8}{|l|}{ Vaginal pH } \\
\hline$<4.5$ & 73 & $10(14)$ & 0.69 (0.34-1.38) & 0.29 & $9(12)$ & $0.61(0.29-1.26)$ & 0.18 \\
\hline 4.5 to $<5.5$ & 68 & $18(26)$ & $1.57(0.89-2.77)$ & 0.11 & $19(28)$ & $1.69(0.96-2.95)$ & 0.06 \\
\hline$\geq 5.5$ & 821 & $153(19)$ & $\operatorname{Ref}(1.00)$ & & $153(19)$ & $\operatorname{Ref}(1.00)$ & \\
\hline \multicolumn{8}{|l|}{ HIV status } \\
\hline Yes & 427 & $109(26)$ & 2.20 (1.58-3.06) & $<0.01$ & $124(29)$ & $3.43(2.43-4.86)$ & $<0.01$ \\
\hline No & 535 & $72(13)$ & $\operatorname{Ref}(1.00)$ & & $57(11)$ & $\operatorname{Ref}(1.00)$ & \\
\hline
\end{tabular}

In age-adjusted analyses comparing HIV-positive and HIVnegative women, the odds ratios (95\% CI; $p$-value) were 2.16 $(1.55-3.01 ;<0.001)$ for prevalent lrHPV; 1.77 (1.23-2.53; 0.001) for prevalent single lrHPV type infection; 3.75 (1.74-8.09; $<0.001)$ for prevalent multiple lrHPV types; $1.53(0.84-2.78 ; 0.16)$ for low-risk group-specific persistence, and 1.41 (0.77-2.60; 0.25$)$ for persistence of specific lrHPV infections. These associations were relatively unchanged in the multivariate models (Table 7). The associations with HPV 44, 54, 66, 70, and 74 at baseline were significantly stronger in HIV-positive compared to HIV-negative women (Figure 4).

For hrHPV infections, the age-adjusted odds ratios (95\% $\mathrm{CI}$; $p$-value) comparing HIV-positive women to HIV-negative women were $3.38(2.39-4.77 ;<0.001)$ for prevalent hrHPV; 2.07 $(1.40-3.08 ;<0.001)$ for prevalent single hrHPV infection; 7.15 $(3.58-14.27 ;<0.001)$ for prevalent multiple hrHPV infection; $4.57(2.38-8.79 ;<0.001)$ for high-risk group-specific persistence; and $4.09(2.05-8.13 ;<0.001)$ for persistence of single hrHPV infections. These associations were relatively unchanged in multivariate models (Table 7). The effect estimates for HPV 16, $18,31,33,35,51,52$, and 58 infections at baseline were significantly higher in HIV-positive compared to HIV-negative women (Figure 4).

\section{DISCUSSION}

Our study describes the patterns of prevalent and persistent lrHPV and hrHPV infections among HIV-negative and HIV-positive women in Nigeria, West Africa. HIV-positive women were more likely to have prevalent, persistent, multiple, group-specific, and type-specific lrHPV and hrHPV infections, compared to HIV-negative women.

Little is known about the epidemiology of HPV infections in Africa. Most studies of cervical HPV infections in SSA have been qualitative studies of prevalent hrHPV genotypes because of their well-described association with cervical cancer. However, given 
TABLE 6 | Association between sociodemographic characteristics and potential risk factors for persistent low-risk and high-risk human papillomavirus (HPV) infections.

\begin{tabular}{|c|c|c|c|c|c|c|c|}
\hline \multirow[t]{2}{*}{ Variable } & \multirow[t]{2}{*}{ Total $n$} & \multicolumn{3}{|c|}{ Persistent low-risk HPV } & \multicolumn{3}{|c|}{ Persistent high-risk HPV } \\
\hline & & $n(\%)$ & Odds ratio $(95 \% \mathrm{Cl})$ & $p$-Value & $n(\%)$ & Odds ratio $(95 \% \mathrm{Cl})$ & $p$-Value \\
\hline \multicolumn{8}{|l|}{ Age, years } \\
\hline$<30$ & 68 & $6(9)$ & $2.36(0.69-8.04)$ & 0.16 & $5(7)$ & $0.83(0.27-2.51)$ & 0.75 \\
\hline 30 to $<45$ & 435 & $39(9)$ & $2.40(0.92-6.23)$ & 0.07 & $47(11)$ & $1.27(0.64-2.54)$ & 0.48 \\
\hline$\geq 45$ & 127 & $5(4)$ & $\operatorname{Ref}(1.00)$ & & $11(9)$ & $\operatorname{Ref}(1.00)$ & \\
\hline \multicolumn{8}{|c|}{ Body mass index, $\mathrm{kg} / \mathrm{m}^{2}$} \\
\hline Normal weight & 195 & $21(11)$ & 1.88 (0.88-4.03) & 0.10 & $24(12)$ & $2.00(0.96-4.13)$ & 0.06 \\
\hline Overweight & 217 & $12(5)$ & $0.91(0.39-2.12)$ & 0.83 & $23(11)$ & $1.68(0.81-3.49)$ & 0.15 \\
\hline Obese, $\geq 30$ & 183 & $11(6)$ & $\operatorname{Ref}(1.00)$ & & $12(7)$ & $\operatorname{Ref}(1.00)$ & \\
\hline \multicolumn{8}{|l|}{ Marital status } \\
\hline Married & 416 & $27(6)$ & 0.57 (0.32-1.03) & 0.06 & $37(9)$ & $0.70(0.41-1.20)$ & 0.19 \\
\hline Not married & 214 & $23(11)$ & $\operatorname{Ref}(1.00)$ & & $26(12)$ & $\operatorname{Ref}(1.00)$ & \\
\hline \multicolumn{8}{|c|}{ Education, years completed } \\
\hline$\leq 6$ years & 74 & $2(3)$ & $0.26(0.05-1.14)$ & 0.07 & $9(12)$ & $1.28(0.53-3.04)$ & 0.57 \\
\hline $7-12$ & 390 & $32(8)$ & $0.83(0.44-1.55)$ & 0.55 & $38(10)$ & $0.99(0.54-1.84)$ & 0.99 \\
\hline$>12$ & 164 & $16(10)$ & $\operatorname{Ref}(1.00)$ & & $16(10)$ & $\operatorname{Ref}(1.00)$ & \\
\hline \multicolumn{8}{|c|}{ Socioeconomic status } \\
\hline Low & 247 & $20(8)$ & $2.11(0.77-5.77)$ & 0.14 & $31(13)$ & $2.09(0.93-4.71)$ & 0.07 \\
\hline Middle & 258 & $25(10)$ & $2.57(0.96-6.89)$ & 0.05 & $24(9)$ & $1.50(0.65-3.44)$ & 0.33 \\
\hline High & 125 & $5(4)$ & Ref (1.00) & & $8(6)$ & $\operatorname{Ref}(1.00)$ & \\
\hline \multicolumn{8}{|c|}{ Age at sexual initiation, years } \\
\hline$\leq 19$ & 295 & $30(10)$ & $2.45(0.99-6.04)$ & 0.05 & $33(11)$ & $1.77(0.82-3.82)$ & 0.14 \\
\hline 19-22 & 187 & $13(7)$ & $1.61(0.59-4.37)$ & 0.34 & $21(11)$ & $1.78(0.79-4.03)$ & 0.16 \\
\hline$>22$ & 136 & $6(4)$ & $\operatorname{Ref}(1.00)$ & & $9(7)$ & $\operatorname{Ref}(1.00)$ & \\
\hline \multicolumn{8}{|c|}{ Total lifetime sex partners } \\
\hline 1 & 157 & $13(8)$ & $1.29(0.54-3.03)$ & 0.55 & $8(5)$ & $0.37(0.16-0.89)$ & 0.02 \\
\hline $2-4$ & 316 & $27(9)$ & $1.33(0.62-2.83)$ & 0.45 & $36(11)$ & $0.90(0.50-1.64)$ & 0.74 \\
\hline$\geq 5$ & 153 & $10(7)$ & $\operatorname{Ref}(1.00)$ & & $19(12)$ & Ref (1.00) & \\
\hline \multicolumn{8}{|l|}{ Douche } \\
\hline Yes & 407 & $39(10)$ & $2.03(1.01-4.05)$ & 0.04 & $46(11)$ & $1.53(0.85-2.75)$ & 0.14 \\
\hline No & 222 & $11(5)$ & $\operatorname{Ref}(1.00)$ & & $17(8)$ & Ref (1.00) & \\
\hline \multicolumn{8}{|l|}{ Vaginal pH } \\
\hline$<4.5$ & 40 & $2(5)$ & $0.58(0.13-2.51)$ & 0.47 & $1(3)$ & $0.23(0.03-1.77)$ & 0.16 \\
\hline 4.5 to $<5.5$ & 42 & $3(7)$ & $0.86(0.25-2.89)$ & 0.80 & $9(21)$ & $2.54(1.15-5.61)$ & 0.02 \\
\hline$\geq 5.5$ & 548 & $45(8)$ & $\operatorname{Ref}(1.00)$ & & $53(10)$ & $\operatorname{Ref}(1.00)$ & \\
\hline \multicolumn{8}{|l|}{ HIV status } \\
\hline Yes & 321 & $31(10)$ & $1.63(0.90-2.95)$ & 0.10 & $51(16)$ & 4.67 (2.44-8.95) & $<0.01$ \\
\hline No & 309 & $19(6)$ & $\operatorname{Ref}(1.00)$ & & $12(4)$ & $\operatorname{Ref}(1.00)$ & \\
\hline
\end{tabular}

that the degree of carcinogenicity of some of the HPV types has not been well elucidated and current studies highlight the marked heterogeneity of HPV infections in this population $(8,22)$, it is necessary to explore associations between type and groupspecific, prevalent and persistent HPV infections, and cervical carcinogenesis in Africa.

Low-risk HPV infections are associated with genital and non-genital warts. Genital warts cause significant physical and psychological morbidities $(10,11)$. They lack specific treatments, are highly recurrent after treatment, and are associated with significant health-care systems' costs. The prevalence of lrHPV infections among HIV-negative women in our study is similar to the $18 \%$ reported among US women (23) but higher than the prevalence of $4 \%$ in South Korean women (24). The prevalence of lrHPV in HIV-positive women in our study (26\%) was lower than the 55\% reported among HIV-positive women in Rwanda (3).

The commonest lrHPV types in our study were unspecified HPV genotypes, HPV 68/73, and HPV 53 in HIV-negative women while unspecified HPV genotypes, HPV 66 and 44 were the commonest types in HIV-positive women. We did not find any significant associations with group or type specific persistent lrHPV. In a study of peri-urban, black South African women, the commonest lrHPV types were HPV 62 (16\%) and 84 (14\%) (25).

HPV 34 and 44 were found in only HIV-positive women in this study probably because their prevalence is low in our study population. Interestingly, we found that lrHPV genotypes 44 and 54, which are associated with external anogenital warts $(9,26)$, and lrHPV genotypes 66 and $70(9)$, which are associated with anogenital precancers and cancers, had significantly higher prevalence in HIV-positive women, compared to HIV-negative women. This lends support to the reports of higher prevalence of anogenital warts and cancers, described in previous studies (27-29).

In contrast to our previous study (8), in this enlarged study population, the commonest prevalent hrHPV types among HIV-negative women were HPV 52, HPV 18, and HPV 35 while HPV 52 and 35 were the commonest persistent infections. Other studies found HPV 52 to be the most prevalent hrHPV in SSA (5, 
TABLE 7 | Risk (odds ratios and 95\% confidence interval) of human papillomavirus (HPV) infections by HIV status.

\begin{tabular}{|c|c|c|c|c|c|c|c|}
\hline & \multirow[t]{2}{*}{$n$ HIV-/HIV+ } & \multicolumn{3}{|c|}{ Age-adjusted model } & \multicolumn{3}{|c|}{ Multivariate model } \\
\hline & & HIV-negative & HIV-positive & $p$-Value & HIV-negative & HIV-positive & $p$-Value \\
\hline \multicolumn{8}{|c|}{ All prevalent HPV infections } \\
\hline Any HPV & $114 / 186$ & 1.00 & $2.81(2.12-3.73)$ & $<0.001$ & 1.00 & $2.84(2.10-3.84)$ & $<0.001$ \\
\hline Low risk & $72 / 109$ & 1.00 & $2.16(1.55-3.01)$ & $<0.001$ & 1.00 & $2.09(1.47-2.97)$ & $<0.001$ \\
\hline High risk & $57 / 124$ & 1.00 & 3.38 (2.39-4.77) & $<0.001$ & 1.00 & $3.38(2.34-4.87)$ & $<0.001$ \\
\hline \multicolumn{8}{|c|}{ Single prevalent HPV } \\
\hline Any HPV & $91 / 100$ & 1.00 & 1.49 (1.08-2.05) & 0.013 & 1.00 & $1.57(1.11-2.20)$ & 0.009 \\
\hline Low risk & 63/82 & 1.00 & 1.77 (1.23-2.53) & 0.001 & 1.00 & 1.75 (1.20-2.56) & 0.003 \\
\hline High risk & $47 / 72$ & 1.00 & $2.07(1.40-3.08)$ & $<0.001$ & 1.00 & $2.12(1.39-3.21)$ & $<0.001$ \\
\hline \multicolumn{8}{|c|}{ Multiple prevalent HPV } \\
\hline Any HPV & $23 / 86$ & 1.00 & 5.47 (3.38-8.85) & $<0.001$ & 1.00 & $5.12(3.11-8.44)$ & $<0.001$ \\
\hline Low risk & $9 / 27$ & 1.00 & 3.75 (1.74-8.09) & $<0.001$ & 1.00 & $3.42(1.54-7.56)$ & 0.002 \\
\hline High risk & $10 / 52$ & 1.00 & $7.15(3.58-14.3)$ & $<0.001$ & 1.00 & $6.93(3.39-14.2)$ & $<0.001$ \\
\hline \multicolumn{8}{|c|}{ All persistent HPV infections ${ }^{a}$} \\
\hline Any HPV & $31 / 76$ & 1.00 & $2.68(1.70-4.22)$ & $<0.001$ & 1.00 & $2.36(1.45-3.82)$ & $<0.001$ \\
\hline Low risk & $19 / 31$ & 1.00 & $1.53(0.84-2.78)$ & 0.16 & 1.00 & $1.26(0.66-2.40)$ & 0.47 \\
\hline High risk & $12 / 51$ & 1.00 & $4.57(2.38-8.79)$ & $<0.001$ & 1.00 & $4.49(2.26-8.91)$ & $<0.001$ \\
\hline \multicolumn{8}{|c|}{ Single persistent HPVa } \\
\hline Any HPV & $30 / 62$ & 1.00 & $2.18(1.36-3.49)$ & 0.001 & 1.00 & $1.88(1.14-3.12)$ & 0.01 \\
\hline Low risk & $19 / 29$ & 1.00 & $1.41(0.77-2.60)$ & 0.25 & 1.00 & $1.17(0.61-2.25)$ & 0.62 \\
\hline High risk & $11 / 42$ & 1.00 & 4.09 (2.05-8.13) & $<0.001$ & 1.00 & $4.13(2.00-8.55)$ & $<0.001$ \\
\hline
\end{tabular}

Table shows ORs and 95\% Cl based on logistic regression models; $n=$ number of women.

Multivariate models were adjusted for age, marital status, and socioeconomic status.

a Multivariate models were adjusted for age, marital status, socioeconomic status, age at sexual initiation, and douching.

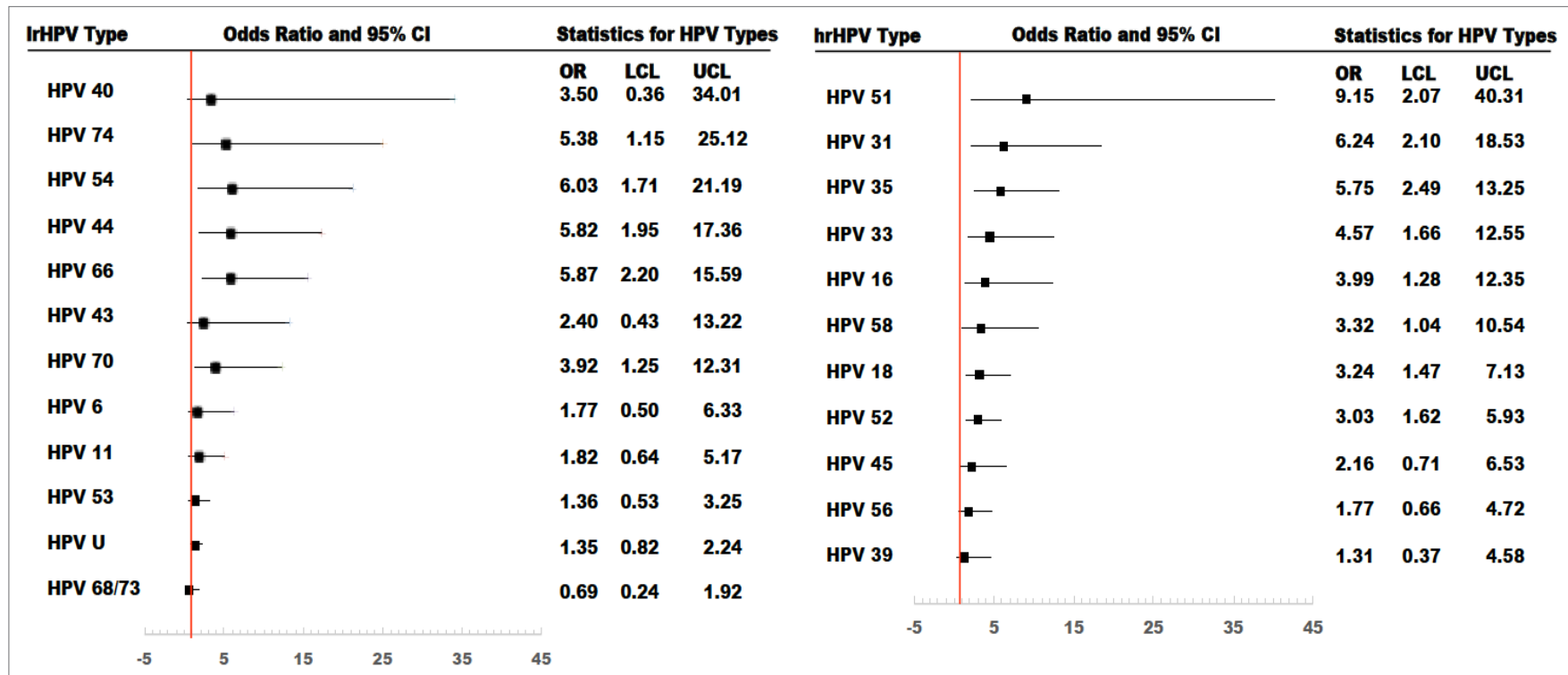

FIGURE 4 | Risk of type-specific prevalent human papillomavirus (HPV) infections among HIV-positive women, compared to HIV-negative women. All models were adjusted. OR, odds ratio; LCL, lower confidenceninterval limit; UCL, upper confidence interval limit.

12, 13). HPV 52, 16, and 18 were the most common hrHPV types observed among HIV-negative Ugandan and Kenyan women $(6,7)$. Among HIV-positive women, the commonest prevalent hrHPV types were HPV 52, 35, and 18, while the commonest persistent hrHPV types were HPV 52, 35, and 31. HPV 52 was also the most prevalent hrHPV genotype observed in a study of HIV-positive Rwandan women (3).
Given the high incidence of cervical cancer in the study population (30), we expected to find more persistent infections among HIV negative and HIV positive woman. Instead, we did not find any HIV-negative woman with persistent HPV 16 or 18 infections while persistent HPV 16 or HPV 18 infections constituted $6.3 \%$ of all persistent infections among HIV-positive women. Non-16/18 HPV types may contribute more to cervical cancer 
development among women in SSA. Many of the cases of persistent hrHPV infections that we observed were with HPV 68/73. Given that HPV 68 is high-risk HPV while HPV 73 is not, studies using technologies that allow differentiation between these two infections should be conducted to examine the association of each HPV type with cervical cancer among women in SSA.

Our findings that the prevalence and persistence of single, multiple, lrHPV and hrHPV infections was higher among HIV-positive compared to HIV-negative women are similar to other reports from SSA $(31,32)$ The increased prevalence and persistence of HPV infection in immunosuppressed individuals suggests that cell-mediated immune response may play an important role in the resolution and control of HPV infection (33). Although some studies have suggested that the association between HIV infection and increased prevalence of HPV infection and disease is related to the immunosuppression seen in HIV infection, there is also some evidence that mechanisms other than immunosuppression, such as direct molecular interactions between HIV and HPV viral genes, may influence the natural history of HPV (34-37). HIV infection may also increase susceptibility to HPV infections and persistence of infection through alterations of the cytokine response to HPV infection in the cervical mucus (38).

The Advisory Committee on Immunization Practices recommended the 9-valent HPV vaccine (Gardasil 9, Merck and Co., Inc.) as one of three HPV vaccines that can be used for routine vaccination (39). This vaccine protects against HPV $6,11,16,18,31,33,45,52$, and 58 , many of which have been observed to be prevalent and persistent in women with single or multiple infections in this study population. Currently, no study has been published on use of the 9-valent HPV vaccine among HIV-positive or negative African women and there are limited data on the response of Africans, regardless of HIV status, to HPV vaccination. The broader coverage provided by the 9-valent vaccine may be sufficient in African populations, regardless of HIV status, and there may be sufficient crossreactivity to ensure protection of HPV types not included in the vaccine. Additional vaccine development and production of vaccines that focus on the commonest HPV types may be helpful in the African population. Further studies on HPV vaccines, immunologic response of Africans to HPV vaccination, and the roles of non-16/18 hrHPV in cervical carcinogenesis in SSA are warranted.

\section{REFERENCES}

1. CDC. Human Papilloma Virus. (2012). Available from: http://www.cdc.gov/ STD/HPV/STDFact-HPV.htm

2. IARCMonographs on the Evaluation of Carcinogenic Risks to Humans: Biological Agents, A Review of Human Carcinogenesis. Lyon, France: International Agency for Research on Cancer (2012).

3. Veldhuijzen NJ, Braunstein SL, Vyankandondera J, Ingabire C, Ntirushwa J, Kestelyn E, et al. The epidemiology of human papillomavirus infection in HIV-positive and HIV-negative high-risk women in Kigali, Rwanda. BMC Infect Dis (2011) 11:333. doi:10.1186/1471-2334-11-333

4. McDonald AC, Tergas AI, Kuhn L, Denny L, Wright TC Jr. Distribution of human papillomavirus genotypes among HIV-positive and HIV-negative women in Cape Town, South Africa. Front Oncol (2014) 4:48. doi:10.3389/ fonc. 2014.00048
Our study has several limitations including lack of inclusion of clinical correlates of HIV infection such as duration of HIV infection, use of anti-retroviral therapy, and viral load or CD4 count in the analyses though these factors may be associated with HPV persistence. However, we excluded HIV-positive women who were severely ill from our study.

In conclusion, we found a higher proportion of prevalent and persistent infections with single and multiple lrHPV and hrHPV genotypes in HIV-positive women, compared to HIV-negative women. We also found that non-16/18 hrHPV genotypes were more common in women with persistent infections regardless of HIV status. Our results support the recommendation of the 9-valent HPV vaccine for routine vaccination, especially in SSA populations where non-16/18 hrHPV types are prevalent and likely to cause persistent infections.

\section{ETHICS STATEMENT}

The study was conducted according to the Nigerian National Code for Health Research Ethics. Ethical approval to conduct this study was obtained from the Institute of Human Virology Nigeria research ethics committee. Written informed consent was obtained from all participants before enrollment in the study, in accordance with the Declaration of Helsinki.

\section{AUTHOR CONTRIBUTIONS}

SA conducted the data analyses, interpreted the data, and drafted the manuscript. CA designed and obtained funding for the study. $\mathrm{OO}$ and AF processed the samples, performed biochemical assays, and collated the laboratory data. ED contributed to the study coordination. SA, OO, AF, ED, RO, and CA contributed to the study implementation and the manuscript. Each author approved the final version of the manuscript and agreed to be accountable for all aspects of the work.

\section{FUNDING}

This work was supported by the UM-Capacity Development for Research in AIDS Associated Malignancy Grant (NIH/ NCI 1D43CA153792-01) and African Collaborative Center for Microbiome and Genomics Research Grant (NIH/NHGRI 1U54HG006947).

5. Gage JC, Ajenifuja KO, Wentzensen NA, Adepiti AC, Eklund C, Reilly M, et al. The age-specific prevalence of human papillomavirus and risk of cytologic abnormalities in rural Nigeria: implications for screen-and-treat strategies. Int J Cancer (2012) 130(9):2111-7. doi:10.1002/ijc.26211

6. Blossom DB, Beigi RH, Farrell JJ, Mackay W, Qadadri B, Brown DR, et al. Human papillomavirus genotypes associated with cervical cytologic abnormalities and HIV infection in Ugandan women. J Med Virol (2007) 79(6):758-65. doi:10.1002/jmv.20817

7. De Vuyst H, Steyaert S, Van Renterghem L, Claeys P, Muchiri L, Sitati S, et al. Distribution of human papillomavirus in a family planning population in Nairobi, Kenya. Sex Transm Dis (2003) 30(2):137-42. doi:10.1097/ 00007435-200302000-00009

8. Akarolo-Anthony SN, Al-Mujtaba M, Famooto AO, Dareng EO, Olaniyan OB, Offiong R, et al. HIV associated high-risk HPV infection among Nigerian women. BMCInfect Dis (2013) 13:521. doi:10.1186/1471-2334-13-521 
9. Cubie HA. Diseases associated with human papillomavirus infection. Virology (2013) 445(1-2):21-34. doi:10.1016/j.virol.2013.06.007

10. Graziottin A, Serafini A. HPV infection in women: psychosexual impact of genital warts and intraepithelial lesions. J Sex Med (2009) 6(3):633-45. doi:10.1111/j.1743-6109.2008.01151.x

11. Maw RD, Reitano M, Roy M. An international survey of patients with genital warts: perceptions regarding treatment and impact on lifestyle. Int J STD AIDS (1998) 9(10):571-8. doi:10.1258/0956462981921143

12. Singh DK, Anastos K, Hoover DR, Burk RD, Shi Q, Ngendahayo L, et al. Human papillomavirus infection and cervical cytology in HIV-infected and HIV-uninfected Rwandan women. J Infect Dis (2009) 199(12):1851-61. doi:10.1086/599123

13. Baay MF, Kjetland EF, Ndhlovu PD, Deschoolmeester V, Mduluza T, Gomo E, et al. Human papillomavirus in a rural community in Zimbabwe: the impact of HIV co-infection on HPV genotype distribution. J Med Virol (2004) 73(3):481-5. doi:10.1002/jmv.20115

14. Chaturvedi AK, Dumestre J, Gaffga AM, Mire KM, Clark RA, Braly PS, et al. Prevalence of human papillomavirus genotypes in women from three clinical settings. J Med Virol (2005) 75(1):105-13. doi:10.1002/jmv. 20244

15. Famooto A, Almujtaba M, Dareng E, Akarolo-Anthony S, Ogbonna C, Offiong R, et al. RPS19 and TYMS SNPs and prevalent high risk human papilloma virus infection in Nigerian women. PLoS One (2013) 8(6):e66930. doi:10.1371/journal.pone.0066930

16. van Hamont D, van Ham MA, Bakkers JM, Massuger LF, Melchers WJ. Evaluation of the SPF10-INNO LiPA human papillomavirus (HPV) genotyping test and the roche linear array HPV genotyping test. J Clin Microbiol (2006) 44(9):3122-9. doi:10.1128/JCM.00517-06

17. Kleter B, van Doorn LJ, Schrauwen L, Molijn A, Sastrowijoto S, ter Schegget J, et al. Development and clinical evaluation of a highly sensitive PCR-reverse hybridization line probe assay for detection and identification of anogenital human papillomavirus. J Clin Microbiol (1999) 37(8):2508-17.

18. Melchers WJ, Bakkers JM, Wang J, de Wilde PC, Boonstra H, Quint WG, et al. Short fragment polymerase chain reaction reverse hybridization line probe assay to detect and genotype a broad spectrum of human papillomavirus types. Clinical evaluation and follow-up. Am J Pathol (1999) 155(5):1473-8. doi:10.1016/S0002-9440(10)65462-4

19. Filmer D, Pritchett LH. Estimating wealth effects without expenditure data - or tears: an application to educational enrollments in states of India. Demography (2001) 38(1):115-32. doi:10.2307/3088292

20. Cox DR, Snell EJ. Analysis of Binary Data. 2nd ed. Boca Raton: Chapman and Hall (1989).

21. Hirji KF. Exact Analysis of Discrete Data. Boca Raton: Chapman and Hall (2006).

22. Akarolo-Anthony SN, Famooto AO, Dareng EO, Olaniyan OB, Offiong R, Wheeler CM, et al. Age-specific prevalence of human papilloma virus infection among Nigerian women. BMC Public Health (2014) 14:656. doi:10.1186/ 1471-2458-14-656

23. Dunne EF, Unger ER, Sternberg M, McQuillan G, Swan DC, Patel SS, et al. Prevalence of HPV infection among females in the United States. JAMA (2007) 297(8):813-9. doi:10.1001/jama.297.8.813

24. Shin HR, Lee DH, Herrero R, et al. Prevalence of human papillomavirus infection in women in Busan, South Korea. Int J Cancer (2003) 103(3):413-21. doi:10.1002/ijc. 10825

25. Van Aardt MC, Dreyer G, Richter KL, Becker P. Human papillomavirus-type distribution in South African women without cytological abnormalities: a peri-urban study. South Afr J Gynaecol Oncol (2013) 5(2):S21-7. doi:10.1080/ 20742835.2013.11441218

26. Muñoz N, Bosch FX, de Sanjosé S, Herrero R, Castellsagué X, Shah KV, et al. Epidemiologic classification of human papillomavirus types associated with cervical cancer. N Engl J Med (2003) 348(6):518-27. doi:10.1056/ NEJMoa021641
27. Minkoff HL, Eisenberger-Matityahu D, Feldman J, Burk R, Clarke L. Prevalence and incidence of gynecologic disorders among women infected with human immunodeficiency virus. Am J Obstet Gynecol (1999) 180(4):824-36. doi:10.1016/S0002-9378(99)70653-8

28. Beutner KR, Reitano MV, Richwald GA, Wiley DJ. External genital warts: report of the American Medical Association Consensus Conference. AMA Expert Panel on External Genital Warts. Clin Infect Dis (1998) 27(4):796-806. doi:10.1086/514964

29. Dandapani SV, Eaton M, Thomas CR Jr, Pagnini PG. HIV-positive anal cancer: an update for the clinician. J Gastrointest Oncol (2010) 1(1):34-44. doi:10.3978/j.issn.2078-6891.2010.005

30. Jedy-Agba E, Curado MP, Ogunbiyi O, Oga E, Fabowale T, Igbinoba F, et al. Cancer incidence in Nigeria: a report from population-based cancer registries. Cancer Epidemiol (2012) 36(5):e271-8. doi:10.1016/j.canep.2012.04.007

31. De Vuyst H, Ndirangu G, Moodley M, Tenet V, Estambale B, Meijer CJ, et al. Prevalence of human papillomavirus in women with invasive cervical carcinoma by HIV status in Kenya and South Africa. Int J Cancer (2012) 131(4):949-55. doi:10.1002/ijc.26470

32. van Aardt MC, Dreyer G, Pienaar HF, Karlsen F, Hovland S, Richter KL, et al. Unique human papillomavirus-type distribution in South African women with invasive cervical cancer and the effect of human immunodeficiency virus infection. Int J Gynecol Cancer (2015) 25(5):919-25. doi:10.1097/ IGC.0000000000000422

33. Scott $M$, Nakagawa $M$, Moscicki AB. Cell-mediated immune response to human papillomavirus infection. Clin Diagn Lab Immunol (2001) 8(2):209-20. doi:10.1128/CDLI.8.2.209-220.2001

34. Arany I, Tyring SK. Systemic immunosuppression by HIV infection influences HPV transcription and thus local immune responses in condyloma acuminatum. Int J STD AIDS (1998) 9(5):268-71. doi:10.1258/0956462981922197

35. Dolei A, Curreli S, Marongiu P, Pierangeli A, Gomes E, Bucci M, et al. Human immunodeficiency virus infection in vitro activates naturally integrated human papillomavirus type 18 and induces synthesis of the L1 capsid protein. J Gen Virol (1999) 80( Pt 11):2937-44. doi:10.1099/0022-1317-80-11-2937

36. Moscicki AB, Ellenberg JH, Vermund SH, Holland CA, Darragh T, Crowley-Nowick PA, et al. Prevalence of and risks for cervical human papillomavirus infection and squamous intraepithelial lesions in adolescent girls: impact of infection with human immunodeficiency virus. Arch Pediatr Adolesc Med (2000) 154(2):127-34. doi:10.1001/archpedi.154.2.127

37. Vernon SD, Hart CE, Reeves WC, Icenogle JP. The HIV-1 tat protein enhances E2-dependent human papillomavirus 16 transcription. Virus Res (1993) 27(2):133-45. doi:10.1016/0168-1702(93)90077-Z

38. Crowley-Nowick PA, Ellenberg JH, Vermund SH, Douglas SD, Holland CA, Moscicki AB. Cytokine profile in genital tract secretions from female adolescents: impact of human immunodeficiency virus, human papillomavirus, and other sexually transmitted pathogens. J Infect Dis (2000) 181(3):939-45. doi:10.1086/315311

39. Petrosky E, Bocchini JA Jr, Hariri S, Chesson H, Curtis CR, Saraiya M, et al. Use of 9-valent human papillomavirus (HPV) vaccine: updated HPV vaccination recommendations of the advisory committee on immunization practices. MMWR Morb Mortal Wkly Rep (2015) 64(11):300-4.

Conflict of Interest Statement: The authors declare that the research was conducted in the absence of any commercial or financial relationships that could be construed as a potential conflict of interest.

Copyright (C) 2017 Adebamowo, Olawande, Famooto, Dareng, Offiong and Adebamowo. This is an open-access article distributed under the terms of the Creative Commons Attribution License (CC BY). The use, distribution or reproduction in other forums is permitted, provided the original author(s) or licensor are credited and that the original publication in this journal is cited, in accordance with accepted academic practice. No use, distribution or reproduction is permitted which does not comply with these terms. 